\title{
RESPOSTAS DO CACAUEIRO À APLICAÇÃO DE N, P E K EM DOIS SOLOS DA AMAZÔNIA BRASILEIRA ${ }^{(\mathbf{1})}$
}

\author{
F. I. O. MORAIS ${ }^{(2)}$
}

\begin{abstract}
RESUMO
A cacauicultura da Amazônia está implantada em solos eutróficos, com predominância da Terra Roxa Estruturada, eem Latossolos ou Podzólicos distróficos, sendo desconhecidas as limitações nutricionais desses solos na fase produtiva do cacaueiro. As respostas do cacaueiro à aplicação de N, P e K foram determinadas em dois experimentos instalados nos municípios de Medicilândia, ao longo da Rodovia Transamazônica, e Benevides, Pará, em solos das unidades Terra Roxa Estrutura eutrófica (TR) e Latossolo Amarelo (LA), respectivamente. As lavouras de cacau do híbrido Sca 6 x Be 10 foram implantadas após o corte e quei ma da mata primária, utilizando-se, como esquema experimental, um fatorial NPK $2^{3}$ com tratamentos adicionais de $\mathbf{P}$. Os resultados obtidos demonstraram que 0 P foi 0 principal nutriente que limitou a produção, provocando incrementos de produtividade $(P<0,01)$ da ordem de $13,7 \%$ (110 kg ha-1) e 44,3\% (214 kg ha-1) nos solos TR e LA, respectivamente, na média do período 1987 a 1993 . O K também aumentou ( $P<0,01)$ o rendimento de amêndoas secas de cacau no solo $L A$, verificando-se, ainda, interações significativas $(P<0,05)$ entre $N$ x K e P x K neste solo. A resposta linear do cacaueiro ao $P$ e o aumento ou diminuição da taxa de infecção de vassoura-de-bruxa ao N, P e K evidenciam a necessidade de novas pesquisas para definir a dosagem econômica de $P$, de $K$ e o efeito da interação dos nutrientes com a enfermidade.
\end{abstract}

Termos de indexação: cacau, adubação, macronutrientes, vassoura-de-bruxa.

\section{SUMMARY: RESPONSES OF CACAO TO N, P AND KON TWO AMAZON BASIN SOILS OF BRAZIL}

Thenew planting areas of cacao in theAmazon basin of Brazil wereestablished on eutrophic soils, especially structured Purple Earth, and on Yellow Latosol or dystrophic podzolic soils. Thenutrient requirements of cacao trees cultivated on thesesoils werestill unknown. The effects of $\mathrm{N}, \mathrm{P}$ and $\mathrm{K}$ on the productivity of cacao weredetermined in two experiments conducted in the counties of Medicilândia, along the Transamazonian Highway, and Benevides, Pará, Brazil.

\footnotetext{
(1) Recebido para publicação em outubro de 1994 e aprovado em novembro de 1997.

(2) Pesquisador da CE PLAC, aposentado, Professor Visitante da Faculdade de Ciências Agrárias do Pará. Rua Tiradentes, 590/701, CEP 66053-330 Belém (PA).
} 


\begin{abstract}
The cacao crop of the hybrid Sca 6 × Be 10 was established on soil units of structured Purple Earth (TR) and Yellow Latosol (LA), after the slash and burn of the natural vegetation. The experimental design was a NPK $2^{3}$ factorial with additional treatments of P. Results showed that $P(P<0,01)$ increased the productivity on TR and LA soils by $13,7 \%\left(110 \mathrm{~kg} \mathrm{ha}^{-1}\right)$ and $44,3 \%\left(214 \mathrm{~kg} \mathrm{ha}^{-1}\right)$, respectively, on average, during theperiod 1987 to 1993 . Potassium $(P<0,01)$ increased yiedd on LA. Significant N $\times$ K and $P \times K$ interactions $(P<0,05)$ on yield were also obtained in this soil. Thelinear response of cacao to $\mathrm{P}$ application and theincrease or decrease in the infection of witches' broom disease due to N, P or K applications suggest the need for additional research to defineeconomically optimum rates of $\mathrm{P}$ and $\mathrm{K}$ and to el ucidatethe effect of the reaction of thesenutrients on witches' broom infections.
\end{abstract}

Index terms: cacao, fertilization, macronutrients, witches' broom.

\section{INTRODUÇÃO}

O plantio do cacaueiro na Amazônia foi iniciado em 1976, conforme acordo cel ebrado entrea Comissão Executiva do Plano da Lavoura Cacaueira (CEPLAC) e a Secretaria de Agricultura do Estado do Pará (SAGRI). Existem, atualmente, cerca de 110.000 hectares plantados, distribuídos, principal mente, nos estados do Pará, Rondônia e Mato Grosso, envol vendo 13.000 produtores rurais e um potencial de produção de 165.000 t de amêndoas secas de cacau, no valor estimado de duzentos milhões de reais.

As áreas demaior desenvol vimento da cacauicultura situam-se ao longo da Rodovia Transamazônica, no Pará, eem Rondônia, ondeocorrem extensas manchas de solos eutróficos, do tipo Terra Roxa Estruturada, e latossolos ou podzólicos distróficos, extremamente pobres em nutrientes (Falesi, 1972; Silva et al., 1973; Neves \& Barbosa, 1982; Morais \& Campos, 1986).

Nos últimos quinze anos, diversos experimentos foram realizados para a obtenção de conhecimentos sobre a capacidade produtiva desses sol os ea resposta do cacaueiro à aplicação de fertilizantes e corretivos (Campos, 1982; Morais \& Campos, 1986; Morais \& Pereira 1986; Morais \& Santos, 1986; Pereira \& Morais, 1987; M orais, 1988). Os dados obtidos na fase inicial de desenvolvimento das plantas no presente experimento foram descritos por Morais \& Pereira (1986) e Morais (1988).
O objetivo deste trabalho foi determinar o efeito dos nutrientes N, P eK na produção de amêndoas secas e na infecção de vassoura-de-bruxa do cacaueiro em sol os da Amazônia.

\section{MATERIAL E MÉTODOS}

O experimento foi desenvol vido em duas plantações de cacau do híbrido Sca 6 × Be 10, estabelecidas em solos das unidades Terra Roxa Estruturada eutrófica (TR) e Latossol oAmarelo (LA), situadas no município de Medicilândia, Rodovia Transamazônica no trecho Altamira Itaituba, $\mathrm{Km} \mathrm{100,} \mathrm{e} \mathrm{no} \mathrm{município} \mathrm{de}$ Benevides, respectivamente, no Pará, após o corte e queima da mata primária. As propriedades físicas e químicas sel ecionadas dos solos (Quadro 1 ) foram analisadas de acordo com o método descrito por Santana et al. (1977), obtidas nas áreas experimentais antes da derrubada da floresta, estão sumariadas no quadro 1 , incluindo os dados de composição mineralógica, extraídos de Morais (1975), e de ferro livre, extraídos de N eves \& Barbosa (1977).

O clima de Medicilândia é caracterizado por um período chuvoso de dezembro a maio, e um período seco, de junho a novembro. O total médio anual de chuvas situa-se em torno de $2.200 \mathrm{~mm}$, com um déficit hídrico médio de $275 \mathrm{~mm}$ no período seco do ano. A insolação média anual é de aproximadamente

Quadro 1. Propriedades químicas, físicas e mineralógicas dos solos(1)

\begin{tabular}{|c|c|c|c|c|c|c|c|c|c|c|c|}
\hline Solo & Profundidade & Argila & C & pH & $\mathbf{P}$ & $\mathrm{Ca}$ & Mg & $\mathbf{K}$ & Al & $\mathrm{CTC}^{(2)}$ & $\mathrm{Fe}_{2} \mathrm{O}_{3}$ \\
\hline & $\mathrm{cm}$ & $-\mathrm{g} \mathrm{k}$ & ${ }^{-1}-$ & & $\mathrm{mg} \mathrm{kg}^{-1}$ & - & 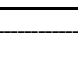 & $\mathrm{nol}_{\mathrm{c}} \mathrm{k}$ & & & $\mathrm{g} \mathrm{kg}^{-1}$ \\
\hline $\operatorname{TR}^{(3)}$ & $\begin{array}{r}0-20 \\
20-40\end{array}$ & $\begin{array}{l}460 \\
600\end{array}$ & $\begin{array}{r}12,1 \\
5,1\end{array}$ & $\begin{array}{l}6,2 \\
6,6\end{array}$ & $\begin{array}{l}3 \\
1\end{array}$ & $\begin{array}{l}57 \\
16\end{array}$ & $\begin{array}{r}17 \\
5\end{array}$ & $\begin{array}{l}5,0 \\
3,5\end{array}$ & $\begin{array}{l}0 \\
0\end{array}$ & $\begin{array}{l}79,4 \\
24,5\end{array}$ & $\begin{array}{l}40 \\
47\end{array}$ \\
\hline $\operatorname{LA}^{(4)}$ & $\begin{array}{r}0-20 \\
20-40\end{array}$ & $\begin{array}{l}210 \\
220\end{array}$ & $\begin{array}{l}7,7 \\
4,7\end{array}$ & $\begin{array}{l}4,8 \\
4,6\end{array}$ & $\begin{array}{l}2 \\
1\end{array}$ & $\begin{array}{l}4 \\
3\end{array}$ & $\begin{array}{l}2 \\
2\end{array}$ & $\begin{array}{l}0,5 \\
0,5\end{array}$ & $\begin{array}{l}9 \\
6\end{array}$ & $\begin{array}{l}15,5 \\
11,5\end{array}$ & $\begin{array}{l}2 \\
3\end{array}$ \\
\hline
\end{tabular}

(1) Minerais da fração argila $(<0,002 \mathrm{~mm})$ do solo TR = caulinita, vermiculita, hematita, goetita, quartzo e tal co, LA =caulinita, gibsita, goetita e quartzo (Morais, 1975). ${ }^{(2)}$ CTC Efetiva. ${ }^{(3)}$ TR = Terra Roxa Estruturada eutrófica. ${ }^{(4)}$ LA = Latossolo Amarelo. 
1.520 horas, sendo a temperatura média do ano de $26^{\circ} \mathrm{C}$, com uma amplitude térmica de $1,1^{\circ} \mathrm{C}$ entre a média dos meses mais secos e chuvosos do ano. O dima de Benevides é semelhante ao de Belém, com um período chuvoso, de dezembro a junho, e um período de menor intensidade de chuvas, de jul ho a novembro. A precipitação pluviométrica anual atinge $2.700 \mathrm{~mm}$, com déficit hídrico médio do período seco de $37 \mathrm{~mm}$, especialmente nos meses de outubro e novembro. A insolação média anual é de 2.360 horas, sendo a tempe ratura média anual de $26,2^{\circ} \mathrm{C}$ (Morais \& Scerne, 1986).

O sombreamento provisório das lavouras de cacau foi de bananeira (Musa spp.), no espaçamento de $3 \times 3 \mathrm{~m}$. O sombreamento permanente foi obtido com o cultivo da eritrina (Erythrina poeppigiana), planta leguminosa, na Terra Roxa, e de gmelina (Gmelina arborea) no Latossolo Amarelo, em Benevides, no espaçamento de $24 \times 24 \mathrm{~m}$. As mudas de cacau foram transplantadas para ocampo em abril de 1982, após seis meses deviveiro, utilizando-seoespaçamento de $3 \times 3 \mathrm{~m}$.

O esquema experimental básico foi um fatorial NPK $2^{3}$, com tratamentos adicionais de fósforo (120 e $240 \mathrm{~kg}$ ha-1 de $\mathrm{P}_{2} \mathrm{O}_{5}$ ), calagem (calcário dolomítico na dosagem de $1 \mathrm{t} \mathrm{ha-1),} \mathrm{uma} \mathrm{mistura} \mathrm{de} \mathrm{micronutrientes}$ ( $2 \mathrm{~kg} \mathrm{ha}^{-1} \mathrm{deB}, 9 \mathrm{~kg} \mathrm{ha}^{-1}$ deZn, $4 \mathrm{~kg} \mathrm{ha} \mathrm{a}^{-1} \mathrm{deFe}, 3 \mathrm{~kg} \mathrm{ha}^{-1} \mathrm{de}$ Cu e de Mo), matéria orgânica (esterco de gado nas dosagens de 1 e $5 \mathrm{t} \mathrm{ha}^{-1}$ ) efracionamento da adubação NPK em três aplicações anuais, com três repetições, de 20 cacaueiros úteis por parcela, em cada local de pesquisa, e bordaduras simples entre parcelas e blocos (M orais, 1988). Fontes dos nutrientes N, P, K foram a uréia, o superfosfato triplo e o cloreto de potássio, respectivamente. A dosagem por planta do fertilizante foi aplicada a lanço, no espaço compreendido entre quatro cacaueiros. As doses de N, $\mathrm{P}_{2} \mathrm{O}_{5}$ e $\mathrm{K}_{2} \mathrm{O}$ foram 0 e $60 \mathrm{~kg} \mathrm{ha}^{-1} \mathrm{ano}^{-1}$. Os tratos culturais foram efetuados de acordo com as recomendações da CE PLAC (Garcia et al., 1985)

Os dados relatados neste trabalho referem-se à parte fatorial e aos tratamentos adicionais de $P$ (Gomes, 1977). Os parâmetros de avaliação foram as alterações verificadas nas propriedades químicas do solo, à profundidade de $0-20 \mathrm{~cm}$, desde a eliminação da floresta até 1991; a produção de amêndoas secas e a perda de frutos por infecção de vassoura-de-bruxa (VB), enfermidade causada pelo fungo Crinipellis perniciosa, na fase produtiva das lavouras de cacau. A porcentagem de frutos perdidos ou infectados com VB foi calculada a partir da fórmula:

$$
\text { VB }(\%)=F i \times 100 / T f p
$$

em que

$\mathrm{Fi}=\mathrm{Frutos}$ perdidos por infecção de vassoura-debruxa; $\mathrm{Tfp}=$ Total de frutos produzidos.

\section{RESULTADOS E DISCUSSÃO}

A figura 1 e o quadro 2 mostram o efeito da aplicação de nutrientes $\mathrm{N}, \mathrm{P}$ e K na produção de amêndoas secas de cacau, considerando o tempo e os solos usados no experimento. A aplicação de P provocou incrementos de produtividade $(P<0,01)$ da ordem de $13,7 \%$ (110 kg ha-1) e 44,3\% (214 kg ha-1) nos sol os TR e LA, respectivamente, na média do período de1987 a 1993. OK aumentou ( $P<0,01)$ a produtividade do cacaueiro no Latossolo Amarelo, verificando-se, ainda, a ocorrência de interações significativas $(\mathrm{P}<0,05)$ entre $\mathrm{P} \times \mathrm{K}$ e N $\times \mathrm{K}$ nesse solo (Quadro 2). Em nenhum solo foi observado efeito do $\mathrm{N}$ sobre o rendimento do cacaueiro, devido, provavel-mente, ao tipo e à intensidade do sombreamento. As árvores utilizadas para sombrear o cacaueiro contribuíram, aparentemente, para incrementar o nível desse elemento no solo pela adição de resíduos orgânicos (folhas, galhos, frutos, etc.) e/ou fixação de $\mathrm{N}$ em nódulos radiculares da eritrina, diminuindo a probabilidade de resposta da cultura à aplicação de fertilizantes nitrogenados. A fixação de N pelas raízes da eritrina foi demonstrada por Santana et al. (1988). A aplicação de $P$ foi também responsável pelo maior crescimento em diâmetro do caul e do cacauei ro no solo LA, na fase inicial do experimento (Morais, 1988). Efeitos do $\mathrm{P}$, do $\mathrm{K}$ e das interações $\mathrm{N} \times$ sombreamento, $\mathrm{P} \times \mathrm{K}$ e $\mathrm{N} \times \mathrm{K}$ na produtividade do cultivo foram também obtidos em outras regiões produtoras decacau, a exemplo da Costa do Marfim (J adin, 1972), da Malásia (Mainstone\& Thong, 1978), do Sul da Bahia (Morais et al., 1978) e de Gana (Ahenkorah et al., 1983).

$\mathrm{O} P$ reduziu o número de frutos infectados pela vassoura-de-bruxa no solo TR $(P<0,05)$ e, em maior intensidade, no solo LA ( $P<0,01)$. Neste último, a aplicação de $\mathrm{N}$ e K contribuiu para aumentar $(P<0,05)$ a porcentagem de frutos infectados pela doença. Acredita-se que o maior crescimento vegetativo do cacaueiro, ocasionado pela adição de $\mathrm{K}$ e, especialmente, de N, produza tecidos tenros mais suscetíveis à infecção da doença, aumentando o potencial de inóculo do fungo, havendo a necessidade de se comprovar essa hipótese. Sabe-se, além disso, que brotações sadias de cacau contêm maiores teores de $\mathrm{Ca}, \mathrm{Mg}, \mathrm{P}$ e $\mathrm{Zn}$ do que aquelas infectadas pela enfermidade (Bastos \& Pereira, 1991), indicando que a nutrição balanceada do cacaueiro pode torná-lo menos suscetível ao ataque da doença.

$\mathrm{Em}$ ambos os solos, observaram-se pequenos aumentos nos val ores de pH eCa + Mg na fase inicial, seguidos de decréscimos para valores próximos ou inferiores aos encontrados originalmente no solo (Figura 3). Tal comportamento foi devido, provavelmente, à adição e lixiviação de cinzas no processo de queima da mata primária (Morais \& Santos, 1986). O alumínio trocável apresentou tendência semelhante, especialmente no Latossolo Amarelo. O P e o K foram os elementos de maior dinâmica, principalmenteoK no soloTerra Roxa (TR). $\mathrm{O}$ teor de $\mathrm{K}$ neste solo variou de $5,0 \mathrm{mmol}_{\mathrm{C}} \mathrm{kg}^{-1}$, em 1981, a 1,0 mmol $\mathrm{kg}^{-1}$, em 1990. O solo TR contém vermicul ita na fração col oidal, havendo a possi bilidade de fixação do $K$ nos espaços entre camadas do mineral ou perdas por lixiviação do elemento contido nas cinzas da quei mada enofertilizante(Quadro 1), considerando o elevadoíndicede preci pitação pluviométrica da região. Tanto a fixação quanto a lixiviação diminuem o teor de K no complexo de troca e na solução do solo. 
TR

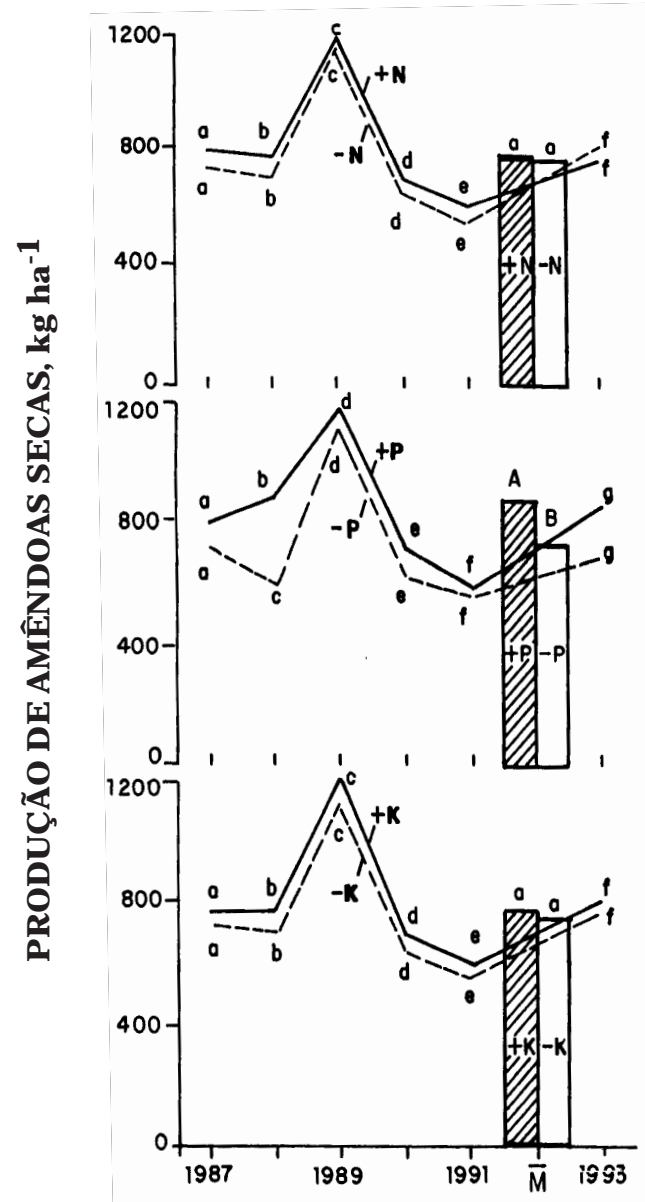

LA

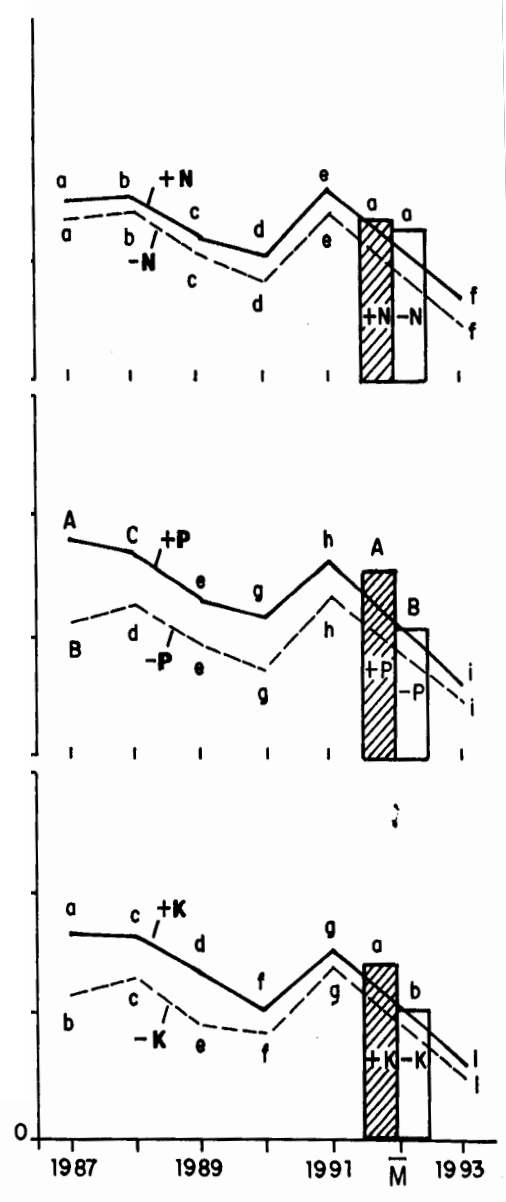

ANO

Figura 1. Produção de amêndoas secas de cacau nos solos TR (Terra Roxa Estruturada) e LA (Latossolo Amarelo), considerando a aplicação de N, P e K. M = produção média do período de 1987 a 1993. Letras diferentes no mesmo ano e na comparação de médias indicam diferenças estatísticas ao nível de $1 \%$ (maiúscula) ou $5 \%$ (minúscula) pelo teste de Duncan.

Quadro 2. Análise de variância dos experimentos relativa à produção de amêndoas secas de cacau

\begin{tabular}{|c|c|c|c|c|c|c|c|}
\hline \multirow{2}{*}{$\begin{array}{c}\text { Fonte } \\
\text { de variação }\end{array}$} & \multirow{2}{*}{ G.L. } & \multicolumn{3}{|c|}{ Terra Roxa - TR } & \multicolumn{3}{|c|}{ Latossolo Amarelo - LA } \\
\hline & & QM & $\mathbf{F}$ & Signif. & QM & $\mathbf{F}$ & Signif. \\
\hline $\mathrm{N}$ & 1 & $39.733,78$ & 1,29 & ns & $92.496,93$ & 3,32 & ns \\
\hline$P$ & 1 & $359.600,10$ & 11,65 & $* *$ & $1.109 .388,00$ & 39,86 & $* *$ \\
\hline K & 1 & $3.402,78$ & 0,11 & ns & $515.042,90$ & 18,50 & $* *$ \\
\hline Bloco & 2 & $31.186,29$ & 1,01 & ns & $59.539,95$ & 2,14 & ns \\
\hline Ano & 6 & $962.750,69$ & 31,19 & $* *$ & $562.427,20$ & 20,21 & $* *$ \\
\hline$N \times P$ & 1 & $22.250,69$ & 0,72 & ns & $5.833,93$ & 0,21 & ns \\
\hline $\mathrm{N} \times \mathrm{K}$ & 1 & $20.688,03$ & 0,67 & ns & $139.207,90$ & 5,00 & * \\
\hline$P \times K$ & 1 & $85.166,70$ & 2,76 & ns & $147.265,90$ & 5,29 & $*$ \\
\hline $\mathrm{N} \times$ ano & 6 & $9.602,43$ & 0,31 & ns & $6.299,38$ & 0,23 & ns \\
\hline $\mathrm{P} \times$ ano & 6 & $49.658,13$ & 1,61 & ns & $41.601,57$ & 1,50 & ns \\
\hline $\mathrm{K} \times$ ano & 6 & $16.250,93$ & 0,53 & ns & $42.377,77$ & 1,52 & ns \\
\hline Resíduo & 135 & $30.865,51$ & - & - & $27.833,42$ & - & - \\
\hline
\end{tabular}


TR

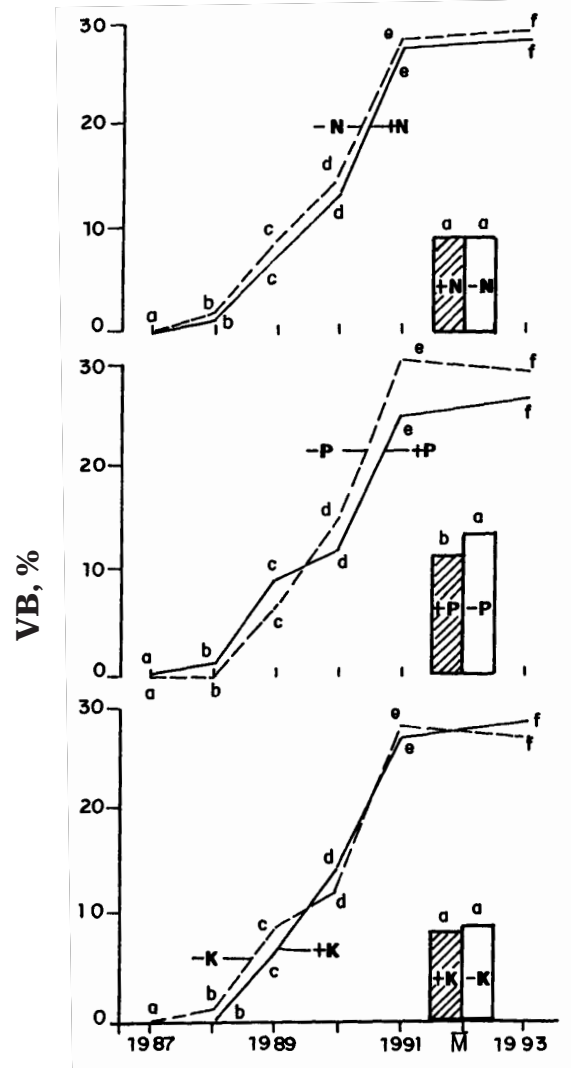

LA
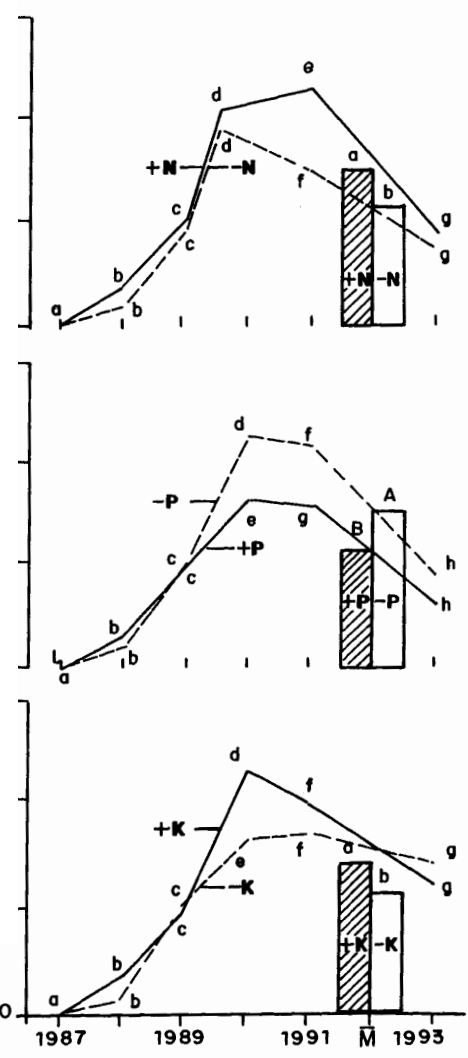

ANO

Figura 2. Efeitos da aplicação de $N, P$ e K na perda de frutos do cacaueiro por infecção de vassoura-debruxa (VB) nos solos TR (Terra Roxa Estruturada) e LA (Latossolo Amarelo). M = produção média do período de 1987 a 1993. Letras diferentes no mesmo ano indicam diferenças estatísticas ao nível de $1 \%$ (maiúscula) ou 5\% (minúscula) pelo teste de Duncan.
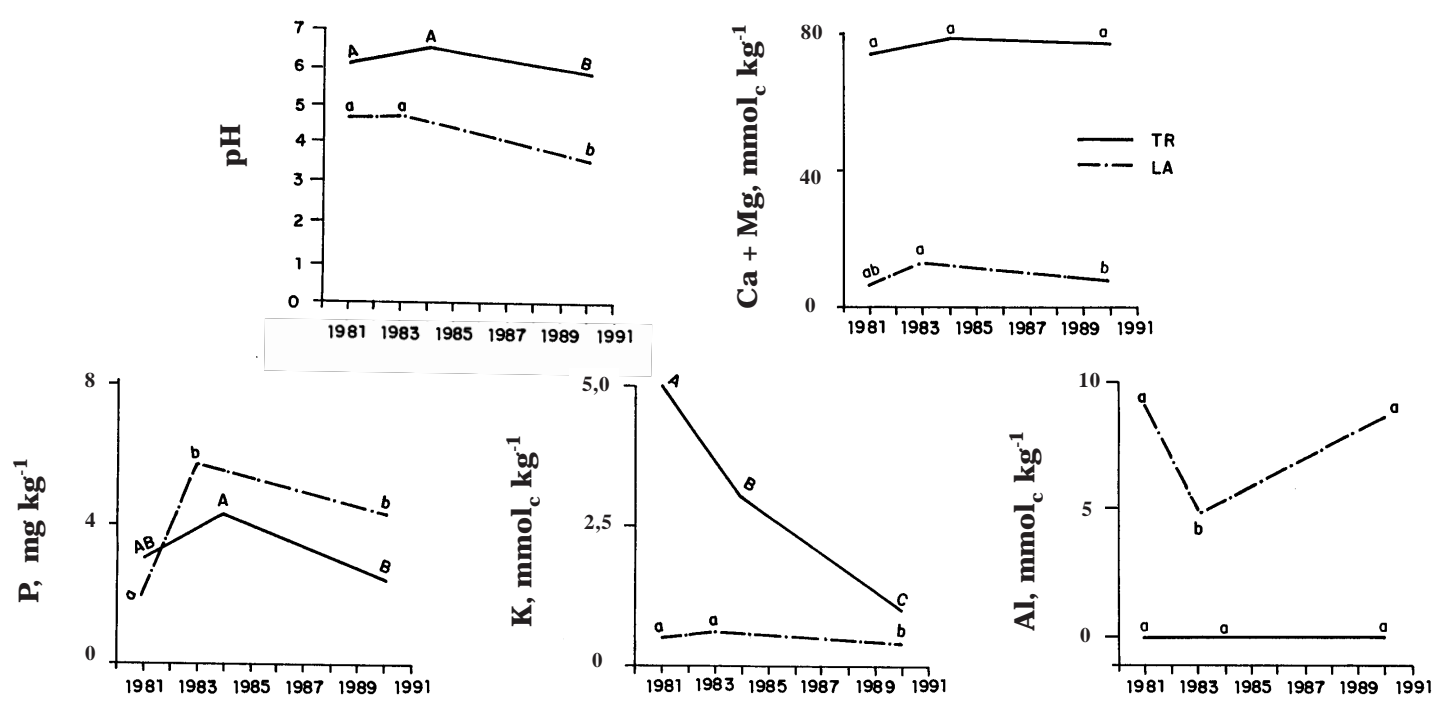

ANO

Figura 3. Modificações nas propriedades químicas dos solos TR (Terra Roxa Estruturada) e LA (Latossolo Amarelo), considerando o tempo de condução do experimento. Amostras dos solos coletadas à profundidade de 0 a $20 \mathrm{~cm}$. Letras diferentes na mesma linha indicam diferenças estatísticas ao nível de $1 \%$ (maiúscula) ou $5 \%$ (minúscula) pelo teste de Duncan. 


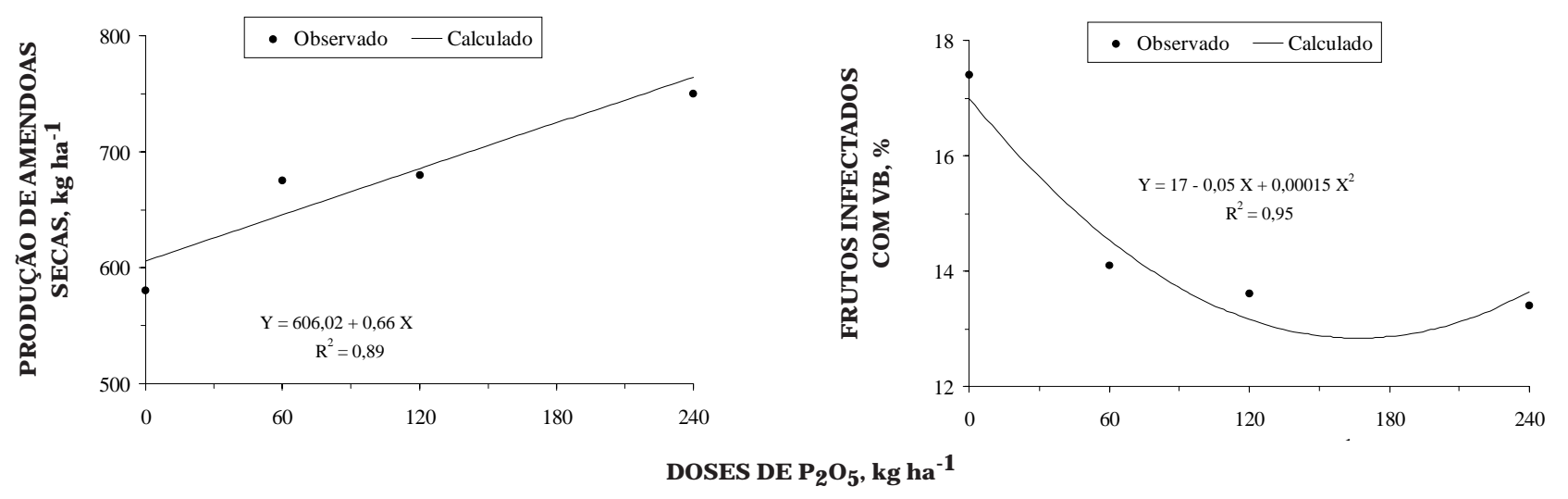

Figura 4. Efeito de doses de $P$ na produção de amêndoas secas (a) e na perda de frutos (b) por infecção de vassoura-de-bruxa (VB). Média dos solos Terra Roxa Estruturada (TR) e Latossolo Amarelo (LA).

O rendimento médio de amêndoas secas de cacau ea perda de frutos por infecção de vassoura-de-bruxa, em função de doses de fósforo, encontram-se na figura 4. Deve-se esclarecer que não houve interação significativa entrea dosagem do nutriente e o tipo de sol o ou ano de condução do experimento. As dosagens de $\mathrm{P}$, de 0 a $240 \mathrm{~kg} \mathrm{ha}^{-1}$ de $\mathrm{P}_{2} \mathrm{O}_{5}$, incrementaram linearmente $\left(Y=606,02+0,66 X, R^{2}=0,89^{* *}\right) \quad a$ produção de amêndoas secas do cacaueiro e reduziram o número de frutos infectados com a enfermidade, sendo a seguinte equação ajustada aos dados obtidos: $Y=17-0,05 X+0,00015 X^{2}\left(R^{2}=0,95^{* *}\right)$. Respostas lineares do cacaueiro à aplicação de $P$ foram também relatadas por Wessel (1971), na África Ocidental, e por Lin \& Mainstone (1982), na Malásia.

\section{CONCLUSÕES}

1. O P foi o principal nutriente que limitou a produção de cacau nos sol os utilizados no experimento. No Latossolo Amarelo, o K proporcionou aumento da produtividade do cacaueiro; o efeito desse el emento foi dependente das doses de $\mathrm{N}$ e $\mathrm{P}$ aplicadas.

2. A resposta do cacauei roà aplicação de $P$ foi linear.

3. O P reduziu o número de frutos atacados pela vassoura-de-bruxa em ambos os solos, enquanto o $\mathrm{N}$ e o K aumentaram a intensidade de incidência dessa enfermidade no Latossolo Amarelo.

\section{LITE RATURA CITADA}

AHENKORAH, Y.; HALM, B.J . \& AKROFI, G.S. Techniques and methods used for identification of suitable soils for growing cacao in Ghana. In: CACAO SOI LS WORKSHOP, 1. BRAZI L, 1977. Techniques and methods for selecting soils suitable for cocoa, Report. Lagos, Nigeria, Cocoa Producers'Alliance, 1983. p. $45-55$.
BASTOS, C.N. \& PEREIRA, G.C. Determinação de níveis de nutrientes em tecidos sadios e infectados de cacaueiros por Crinipellis perniciosa. Belém, CEPLAC, 1991. 86p. (Informe de Pesquisa)

CAMPOS, A.X. Avaliação da fertilidade de solos cacaueiros da Amazônia. Belém, CE PLAC, 1982. 17p. (ComunicadoTécnico, 22)

FALESI, I.C. O estado atual dos conhecimentos sobre os solos da Amazônia brasileira. In: INSTITUTO DE PESQUISAS E EXPERIMENTAÇÃO AGROPECUÁRIAS DO NORTE, ZoneamentoAgrícola da Amazônia. Belém, I PEAN, 1972. p.1767.

GARCIA, J.J.S.; MORAIS, F.I.O.; ALMEIDA, L.C. \& DIAS, J.C. Sistema de produção do cacaueiro na Amazônia Brasileira. Belém, CEPLAC, 1985. 117p.

GOMES, F.P. Curso de estatística experimental. Editora Nobel, São Paulo, 1977. 430p.

J ADIN, P. Study of the mineral fertilization of cocoa trees on the I vory Coast from a "soil diagnosis". Café, Cacao, The, 16:204218, 1972.

LIN, A.M. \& MAINSTONE, B.J . Phosphate requirements of cocoa on Malaysian Inland soils. Kuala Lumpur, The Malaysian Society of Soil Science, 1982. p.365-381.

MAINSTONE, B.J . \& THONG, K.C. Fertilizer response over 6 years from preplanting monocrop cocoa on a Bongor Soil series. Preprint from the International Conference on Cacao and Coconuts. Kuala Lumpur, 1978. 20p.

MORAIS, F.I.O. Charge characteristics and ion exchange equilibria in soils from the humid tropics of Brazil. Riverside, CA, University of California, 1975. 100p. (Ph.D. Dissertation)

MORAIS, F.I.O. Efeito de fertilizantes e corretivos no crescimento e produção do cacaueiro em solos da Amazônia Brasileira. In: CONFERÊNCIA INTERNACIONAL DE PESQUISAS EM CACAU, 10, São Domingo, República Dominicana, 1987. Proceedings, England, Cocoa Producers' Alliance, 1988. p.247251.

MORAIS, F.I.O. \& CAMPOS, A.X. Estado nutricional e produtividade de solos ocupados com cacau na Amazônia Brasileira. In: SIMPÓSIO DO TRÓPICO ÚMIDO, 1. Belém, PA, 1984. Anais. Brasília, EMBRAPA, 1986. p.457-465. 
MORAIS, F.I.O. \& PEREIRA, G.C. Respostas do cacaueiro a aplicação de fertilizantes e corretivos nas condições da Amazônia Brasileira. I. Crescimento e produção inicial. R. Theobroma, 16:65-73, 1986.

MORAIS, F.I.O. \& SANTOS, M.M. Efeitos do sistema de implantação do cacauei ro nas propriedades químicas de sol os da Amazônia brasileira. R. Bras. Ci. Solo, 10:67-70, 1986.

MORAIS, F.I.O.; SANTANA, M.B.M. \& CHEPOTE, R.E. Respostas do cacaueiro ao nitrogênio, fósforo e potássio em solos da região cacaueira da Bahia, Brasil. R. Theobroma, 8:31-41, 1978.

MORAIS, F.I.O. \& SCERNE, R.C. Aspectos climáticos dos pólos cacaueiros da Amazônia Brasileira. In: SIMPÓSIO DO TRÓPICO ÚMIDO, 1., Belém, 1984. Anais. Brasília, EMBRAPA, 1986. p.489-496.

NEVES, A.D. \& BARBOSA, R.C.M. Solos representativos dos pólos cacaueiros da Amazônia. Belém, CEPLAC, 1982. 13p. (Comunicado Técnico Especial, 4)
PEREIRA, G.C. \& MORAIS, F.I .O. Variabilidade química da camada superficial de solos dos pólos cacaueiros da Amazônia. R. Theobroma, 17:143-151, 1987.

SANTANA, M.B.M.; PEREIRA, G.C. \& MORAIS, F.I.O. Métodos de análise de solos, plantas e água utilizados no laboratório do Setor de Fertilidade de Sol os do CEPEC. I lhéus, Centro de Pesquisas do Cacau, 1977. 28p.

SANTANA, M.B.M.; CABALA ROSAND, F.P. \& SERÓDIO, M.H. Reciclagem de nutrientes em agrossistemas de cacau. In: CONFERÊNCIA INTERNACIONAL DE PESQUISAS EM CACAU, 10, São Domingo, República Dominicana, 1987. Proceedings. England, Cocoa Producers'Alliance, 1988. p.233237.

SILVA. L.F.; CARVALHO FILHO, R. \& SANTANA, M.B.M. Solos do projeto Ouro Preto. Ilhéus, CEPLAC, 1973. 31p. (Boletim Técnico, 23)

WESSEL, M. Fertilizer requirements of cacao (Theobroma cacao L.) in South Western Nigeria. Koninklijk, Institut Voor de Tropen, 1971. 104p. 